\title{
Text Channels to Identify and Forestall Hostile Hindi Substances via Online Media
}

\author{
Mr. Pradeep N. Fale ${ }^{1}$, Dr. Krishan Kumar Goyal ${ }^{2}$ \\ ${ }^{1}$ Research Scholar, Department of Computer Science and Engineering, Bhagwant University \\ Ajmer, Rajasthan, India. \\ pradeep.fale@gmail.com \\ ${ }^{2}$ Dean, Faculty of Computer Application, RBSMTC, Agra, India. \\ kkgoyal@gmail.com \\ *Corresponding Author: Mr. Pradeep N. Fale
}

\begin{abstract}
Individual to individual correspondence objections are very renowned these days. With over $70 \%$ of the general population using relational connection objections satisfactorily in India, Hindi correspondence is the most notable. More than $80 \%$ of Indians use Hindi as a technique for correspondence. Websites like Facebook, Twitter, Linkedin, Instagram and WhatsApp have been utilized by people and particularly by teens today. Through individuals-to-individuals like Twitter, Facebook, and so on, the sound of content is shared. Finally, these structures ended up being more many-sided in addressing the necessities of customers to look at their step by step undertakings, feelings and evaluations by posting clear (information) with their associates. Reliably, huge data is shared at different levels of online media. In this article, we will present and discuss thoroughly the execution of message channels for the Hindi language to recognize and prevent harsh substances in relational associations.
\end{abstract}

Keywords: Social Engineering, Cyber Teasing, FB, WhatsApp, Twitter, Pornography, Obscene Content.

\section{Introduction}

Until then, internet customers from all over the world are using and accessing online media groups and welcoming Affiliate Associations (SNS) as the centrepiece of their association, joint relationship effort, information movement and sharing information within associations. Therefore, this review will attempt to use a modified screening methodology that generally channels the objectionable substance and expresses the substance that is dispersed during the long arrival in the released pairing regions.

The proposed approach will be precisely of substance as texts. In this assessment, an improved SNS model is developed for young adult electronic clients, who will be productive 
for their Internet-based security. In this strategy, security is given to the relaxed relationship by applying a filtering tool of the shared substance on the person at the stage of individual matching. The standard basis is essentially the message and the substance that for much of the time carried on in these regions. The model is at an extremely basic level dependent on text-based filtering.

We are building a dynamic Dataset of the words which will eventually be a part of our dictionary that we are building to strengthen our recognition and detection model.

The Algorithm we improved is Naive Bayes Classification. We have modified the algorithm to extend its capabilities to increase the performance index for the application

\section{Review of Work Already Done on the Subject}

Arushi Sharma, Anubha kabra, Minni Jain [1], 2021, the first test studies 'moh' mapped text's overall performance with classical device learning models and shows an average growth of $13 \%$ in $\mathrm{f} 1$ rankings. The second compares the proposed paintings' rankings with the ones of the baseline fashions and shows an upward thrust in overall performance by $6 \%$. Ultimately, the $1 / 3$ compares the proposed 'moh' technique with numerous data simulations using the existing transliteration library. Right here, 'moh' outperforms the relaxation by $15 \%$

Sreelakshmi o k, Premjith b, Soman P [2], 2019,technique uses fb's pre-trained phrase embedding library, fast text to symbolize 10000 records samples accumulated from one of a kind sources as hate and non-hate. The performance of the proposed method is compared with word2vec and doc2vec capabilities and its miles found that fast text functions gave better function representation with support vector device (svm)-radial foundation function (rbf) classifier. The paper also provides a perception to the researchers operating subject of code-blended facts that character stage features provide best result for code-mixed information.

Aya Elouali, Zakaria Elberrichi, Nadia Elouali [3],2019 taking advantages of the benefits of neural networks, classifies tweets written in seven exceptional languages (and even those that contains more than one language at the identical time) to hate speech or non hate speech. We used convolution neural networks ( $\mathrm{cnn}$ ) and person stage illustration. We achieved numerous experiments for you to regulate the parameters in line with our case observe. Our nice results have been (in terms of accuracy) zero. 8893 for a dataset containing 5 languages and 0.8300 for a dataset of 7 languages. Our version solves properly the trouble of hate speech on twitter and its results are, as compared to the country of the artwork, more than first-class.

Raghav kapoor, Yaman kumar, kshitij Rajput, Rajiv Shah [13], 2019, e. We apply transfer gaining knowledge of and make a lstm primarily based version for hate speech type. This version surpasses the performance shown by using the cutting-edge first-rate fashions to establish itself because the contemporary in the unexplored domain of hinglish offensive text class. 
Shivang Chopra, Ramit Sawhney, Puneet Mathur, Rajiv Ratn Shah [14], 2020. A novel bias removal set of rules became proposed to mitigate any gift bias from the model, thereby rendering a fair classification structure.

T. Y. S. S. Santosh, okay. V. S. Aravind [15], 2019, we want to ensure their privacy at the same time as seeking to guard them. On the way to acquire this, we've placed a filtering machine on the server facet that filters the messages and best lets in non-abusive content material to reach the recipient. If any abusive content material is detected, the message is sent to the parents of the sender as well as the receiver. This way, dad and mom ought no longer to be troubled to check all of the messages in their toddler and hinder their privateness

\section{Proposed Work}

With this cyberbullying detection system in social engineering, it is important to indicate the level in order to basically do this assessment, since it is a cyberbullying post-mining system based on text, image and video, of course you need to type of text or language that can be used. This structure is needed to quantify this structure until the focus on the English language is easily developed with an appropriate and formal text. Later in this investigation, Hindi language is accented with a short and relaxed design and consolidated text that educates the audience. The cyberbullying media is trapped in the system. The consideration will be on the tweets posted by customers on SNS and take on customer slogans for planning comments to close the cyberbullying event, the type of cyberbullying and setbacks, and their region. We have compiled an immense variety of over 10,000 oppressive Hindi words that prepared our model. This allows our framework to handle the Hindi word recognition problem. We have put together a compromised app that uses the Chrome extension component to detect abuse on the web. Our software helps us build an abuse management ecosystem. Our code base helps us to extend the software.

To the next:

Chrome extension

Internet Explorer

Mozzila Firefox

Android application

Desktop application 


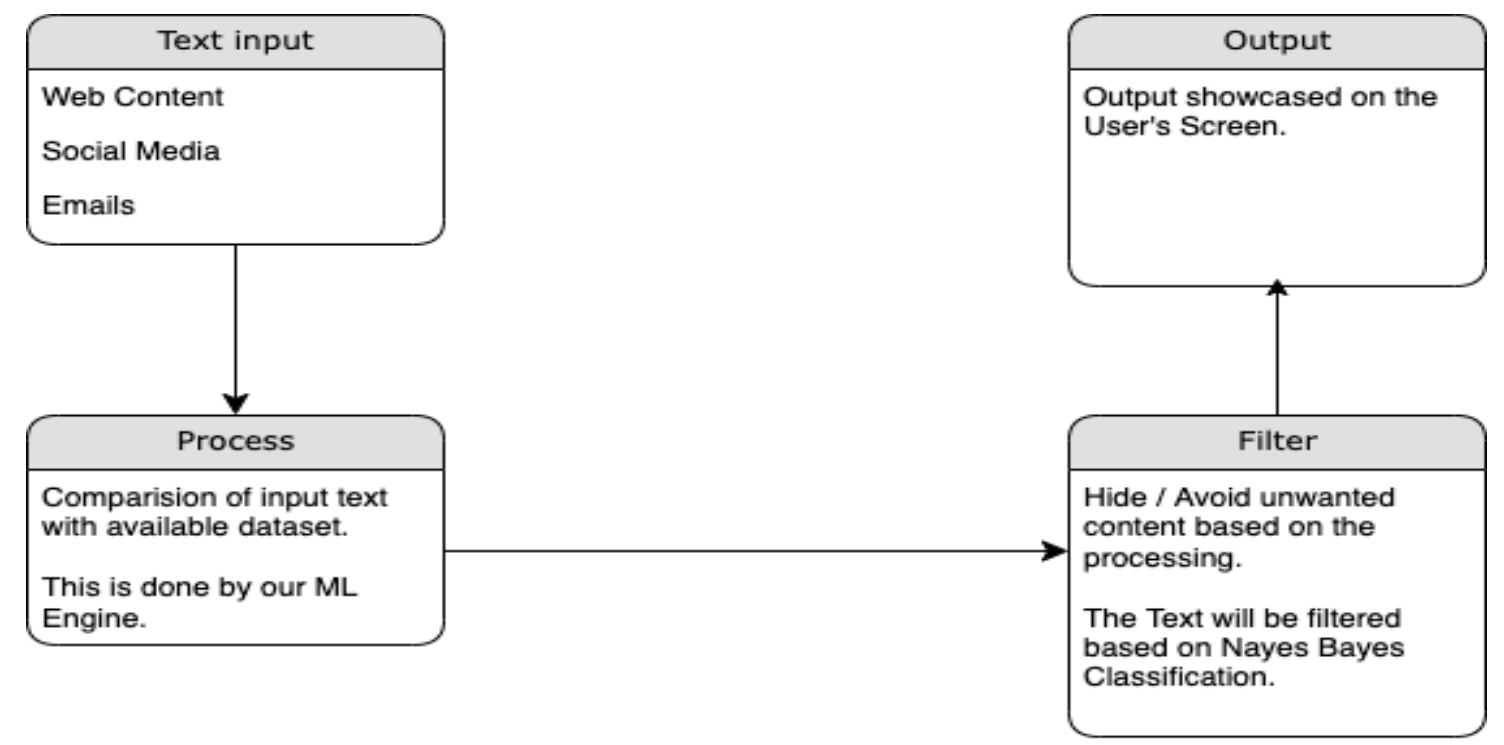

Figure 1. Diagram for Model of Textual Filter.

The Above Diagram showcases the detailed process of our filtering mechanism. The four primary steps involved are:

1. Text Input

2. Process

3. Filter

4. Output

\subsection{Censor Mode}

Censor mode uses movements to hide enemy words by disguising them in a specific style. Here is an overview of the options and an explanation of each. a selected placeholder (default: *) Protect last letter As with the previous selection, with the exception of this, the last letter of a word / phrase is protected Blue pencil mark Specify the person with whom the letters are to be obscured (default: *) Fixed Length Set an appropriate length for the words covered by the channel. Assuming no fixed length is chosen, the word will have the first length

This could help figure out what the word was (default: not the correct length).

\subsection{Replacement Mode}

In substitution mode, hostile words are replaced with a less hostile equivalent. In this mode, a large part of the arrangement you make is on the Words tab, where you customize your summary of words / phrases and what you might want to replace them with. Preserve case. Preserves the first instance of the checked word. (Default: Authorized). Highlight Subtitled Words Highlight subtitled words by surrounding them with [what more]. Standard Substitution this is used as a substitute for words that I don't have a substitute phrase for 


\subsection{Eliminate Mode}

At the point when chosen, the hostile word will essentially be eliminated totally from the page, with no marker that it was ever there. Note that this can now and then prompt peculiar sentences.

\section{Algorithms Reviewed}

\section{A. KSOM (Kohonen Self-Organizing Neural Network)}

In this for seeing texts the philosophy prevalently reliant upon watchword planning and bits of knowledge. Frequencies are incorporated with which expressions appear in a text. Frequencies are used as I/p to the KSOM. Ensuring learning, KSOM (Kohonen SelfOrganizing Neural Network) used to choose unfriendly texts [4]. Lee et al count the frequencies with which watchwords appear in a text.

\section{Bayes Classifier for Discrete Texts}

The semantic relationship in discrete texts can be dismissed, so the frequencies with which the expressions appear in discrete texts are used as the arrangements for affirmation. Hu at al use the Bayes classifier to orchestrate discrete texts, As the Bayes classifier yields probabilities that a text has a spot with the arrangements rather than a particular result that the text has a spot with a class, and the probabilities are used for the blend of texts and pictures.

\section{BOW (Bag-of-Words) Model}

The pack-of-words model is used in NLP and IR [11]. In this model, a message (like a sentence or a chronicle) is tended to as the pack (multiset) of its words, excusing accentuation and even word demand yet keeping collection [11]. The bag-of-words model is by and large used in techniques for record course of action, where the (repeat of) occasion of each word is used as a component for setting up a classifier.

\section{E. Text Mining}

Implementing text mining techniques to separate web-based data requires the following phases [10]: • information gathering and preprocessing, $\bullet$ include extraction, and • clustering. , as follows: 1) Extraction of features at the message level Most studies of hostile substance detection eliminate two types of arrangements: Lexical and Syntactic Parts [10] a) Lexical Features Lexical components treat each word and articulation as a component. Explicit expressions and their frequencies are routinely used to address the language model. The strategies used in this approach are BagsofWords (BOW) and Ngram. Before the role decision equipping an analyzer can help make an effort not to select disjointed phrases as components to detect repulsion. 


\section{Need of the Research Work}

With the coming of this Cyberbullying discovery and arrangement framework in SNS, it will assist the specialists with checking, managing or if nothing else declines the pestering episodes on the internet in India. With the execution of this framework, this will likewise assist with rising the Cyberbullying mindfulness among the SNS clients, and posting the tweets capably in the web-based media, as posting disturbing tweets are unlawful and menaces can be indicted under the Computer Crime. 1) Protect youngsters from Cyberbullying.

2) People know about Cyberbullying.

3) Secure your Social Engineering from deluding text, pictures and video.

\section{Detailed Algorithmic Analysis}

\subsection{Naive Bayes Classification}

It is a characterization strategy dependent on Bayes' theorem with the assumption of freedom between the indicators. Simply put, a Naive Bayesian classifier accepts that the presence of a particular element in one class happens to be due to the presence of another component. A method for determining the inverse probability $\mathrm{P}(\mathrm{c} \mid \mathrm{x})$ from $\mathrm{P}(\mathrm{c}), \mathrm{P}(\mathrm{x})$ and $\mathrm{P}(\mathrm{x} \mid \mathrm{c})$. Check the following situation:

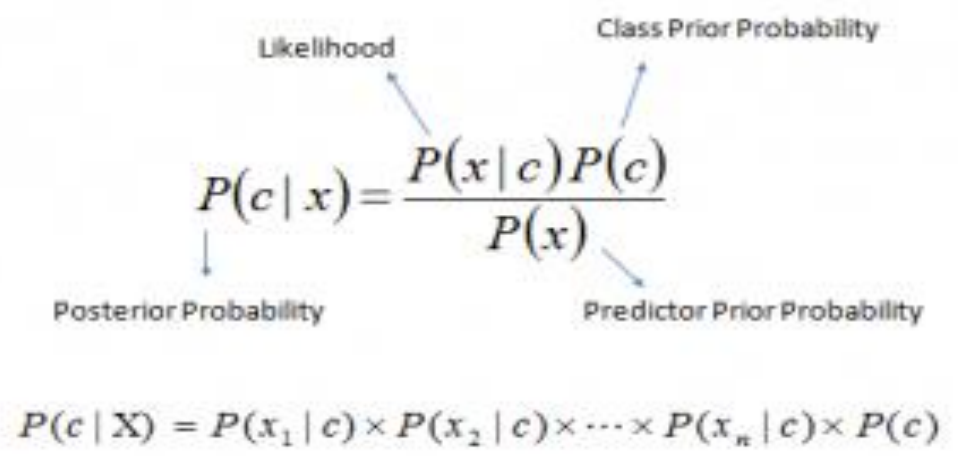

$\mathrm{P}(\mathrm{c} \mid \mathrm{x})$ is the inverse probability of class (c, target) for a given indicator ( $\mathrm{x}$, attributes). $\mathrm{P}(\mathrm{c})$ is the a priori probability of the class. $\mathrm{P}(\mathrm{x} \mid \mathrm{c})$ is the probability that corresponds to the probability of the indicator for a given class. $\mathrm{P}(\mathrm{x})$ is the previous probability of the indicator. Gullible Bayesian classifiers input discrete factors and produce a probability score for each competing class. The expected class grade is the name of the class with the highest probability score. The class grade probabilities depend on the observed qualities. Each element is considered to be autonomous. The probability values of the class names are determined by Bayes' law:

$$
P(C \mid A)=\frac{P(A \cap C)}{P(A)}=\frac{P(A \mid C) P(C)}{P(A)}
$$


Actually, the Bayes' Law computes the restrictive likelihood $\mathrm{P}(\mathrm{C} \mid \mathrm{A})$, addressing the likelihood of $\mathrm{C}$ given that $\mathrm{An}$ is noticed. In this recipe, $\mathrm{C}$ addresses the class name from a bunch of accessible $\mathrm{n}$ classes, and An addresses a perception with $\mathrm{m}$ ascribes:

Thus, for every perception A, we'll to process probabilities for each class mark $\mathrm{I}=1,2, \ldots, \mathrm{n}$ :

$$
P\left(G_{i} \mid A\right)=\frac{P\left(a_{1}, a_{2}, \ldots, a_{m}, G_{i}\right) P\left(G_{i}\right)}{P\left(a_{1}, a_{2}, \ldots, a_{m}\right)}
$$

Really at that time would we be able to settle on the anticipated class $\mathrm{C}$, with the biggest likelihood score $\mathrm{P}\left(\mathrm{C}_{-} \mathrm{i} \mid \mathrm{A}\right)$. We'll apply the accompanying stunts to work on the estimations: Since each characteristic is restrictively free, we can straightforwardly duplicate contingent probabilities of unmistakable traits:

$$
P\left(a_{1}, a_{2}, \ldots, a_{m} \mid O_{i}\right)=\prod_{j=1}^{m} P\left(a_{j} \mid O_{i}\right)
$$

The likelihood $\mathrm{P}(\mathrm{A})$ in the denominator part is steady. Henceforth, we can overlook it when contrasting the scores for various classes.

Subsequently, we can think about the yield of the worked on equation to settle on the class name:

$$
P\left(C_{i} \mid A\right) \propto\left(\prod_{j=1}^{m} P\left(a_{j} \mid C_{i}\right)\right) P\left(C_{i}\right)
$$

The class $\backslash$ boldsymbol $\{\mathrm{C}\}_{-}\{$Vboldsymbol $\{\mathrm{i}\}\}$ with the greatest likelihood is picked to be the anticipated class. As such, we say perception A has a place with class Ci.

The legitimacy of the equation we use to register the class probabilities depends on the contingent autonomy supposition. In the event that two provisions encode a similar data, the Naive Bayes classifier will twofold count their impact and arrive at an off-base resolution.

Graph 1: A Detailed Heatmap and Cluster Map comparison with minimum attributes.

Shown in the below graph, we have had the following results in the below graph. We have showcased the detailed comparison with the attributes Length, $m$ height, Fnocing and mage35. The Results and Data weights are showcased in the X-Axis and the values indexes are showcased in the Y-Axis.

The Red marked is the Cluster Map and Blue mapped is the Heatmap. The Heatmap showcases that the application and model of the input values given to the system are dynamic and can be changed as per the model's highest given attribute values; this significantly improves the model performance and gives us the right results. 
The Cluster map is the simple classification logic we have created to map down the Values between two attributes.

Finally, we conclude that

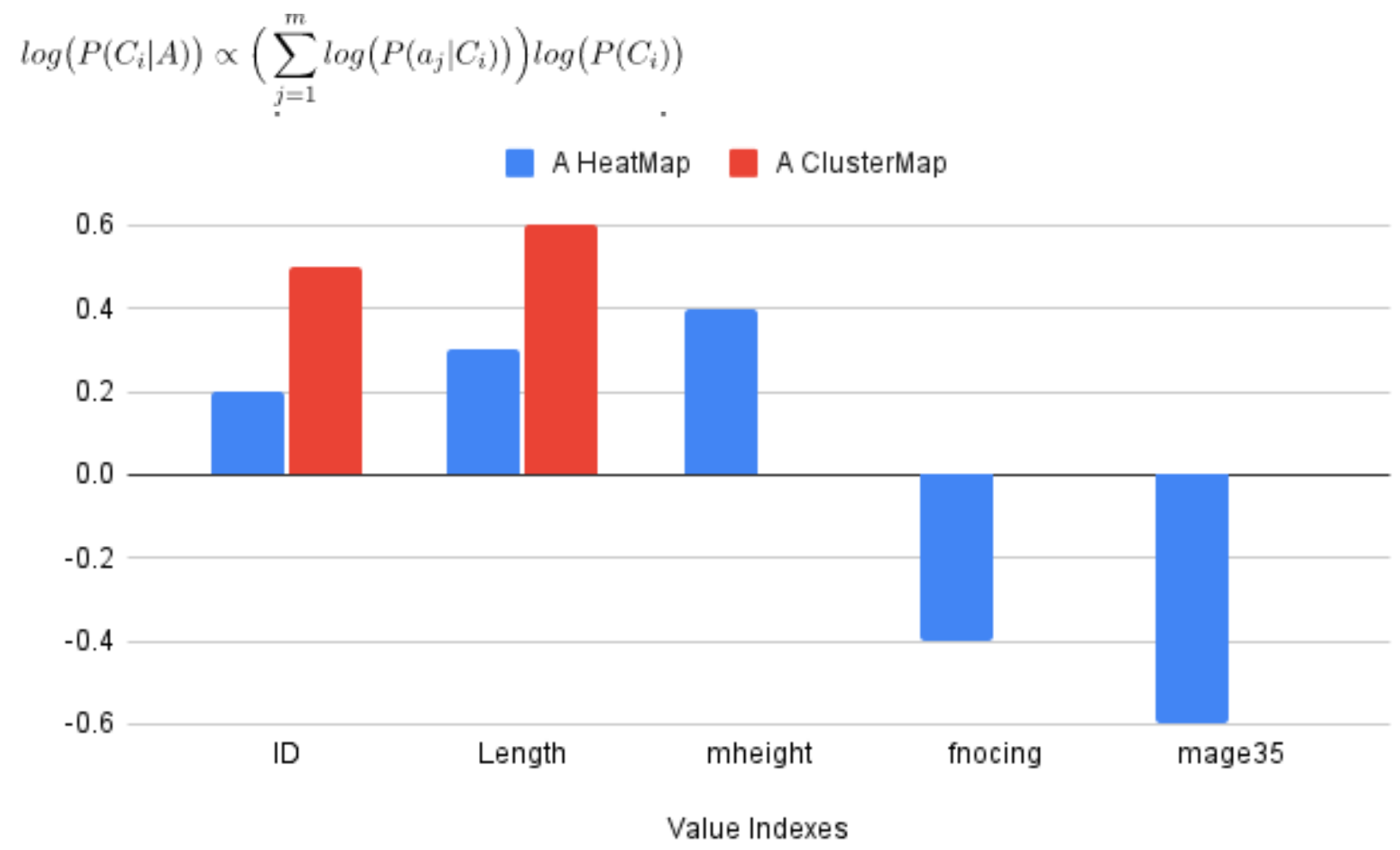

Graph 1: HeatMap and Cluster Map of Naive Bayes Classification

\section{Result}

\begin{tabular}{|l|l|l|}
\hline Value Indexes & A HeatMap & A ClusterMap \\
\hline ID & 0.2 & 0.5 \\
\hline Length & 0.3 & 0.6 \\
\hline mheight & 0.4 & \\
\hline fnocing & -0.4 & \\
\hline mage35 & -0.6 & \\
\hline
\end{tabular}

Table 1: Value Indexes/Attributes comparison between HeatMap and ClusterMap. 
In the above table, we are getting the results of the value indexes in the heatmap and clustermap. The heatmap showcases the detailed analysis of the whole value vs the attributes. Through, we get that more the quality data we feed into the system, the occurrence and stability of the system is more. We get better and accurate results based on the HeatMap and ClusterMap Values.

\section{Conclusion}

With this article, we want to provide a tool that enables people's privacy by filtering obscene language on the internet. In this article, we modified the Naive Bayesian classification model by improving the algorithm's performance and speed. It has been modified to improve the performance of our project. We used a bag of words to contain our libraries which contain all of the offensive / obscene words. This BoW is used as a source to recognize the words online and then the system filter our self-learning algorithm learns from results and existing words on the Internet and then improves the model in order to further improve the recognition quality.

\section{Acknowledgment}

I would like to express my deep gratitude to Dr. Krishan Kumar Goyal my research Supervisor, for their patient guidance, enthusiastic encouragement and useful critiques of this research work.

Finally, I wish to thank my parents for their support and encouragement throughout my study.

\section{References}

\subsection{Journal Article}

[1] Arushi Sharma, Anubha Kabra, Minni Jain, "Ceasing hate with MoH: Hate Speech Detection in Hindi-English code-switched language", Information Processing and Management, 59 (2022) 102760.

[2] Sreelakshmi k, Premjith B. Soman K.P, "Detection of Hate Speech Text in Hindi-English Code-mixed Data", Procedia Computer Science 171 (2020) 737-744.

[3] Aya Elouali, Zakaria Elberrichi, Nadia Elouali, "Hate Speech Detection on Multilingual Twitter Using Convolutional Neural Networks", Revue d' Intelligence Artificielle Vol. 34, No. 1,(2020), pp. 81-88.

[4] Dhruv Mehta, Neelam Gujar, Preet R. Shah, Prof. Stevina Dias, "Co-existence of Privacy and Security of Minors on Social Networks", International Journal for Research in Applied Science \& Engineering Technology (IJRASET), Volume 8 Issue XII Dec (2020), pp 667671.

[5] Anita Kumari Singh, Shashi Mogalla, "AUTOMATIC IDENTIFICATION OF NEWS 
TWEETS ON TWITTER", International Journal of Computer Engineering \& Technology (IJCET) Volume 9, Issue 4, July-August (2018), pp. 140-147.

[6] Neeraj Vashistha , Arkaitz Zubiaga, "Online Multilingual Hate Speech Detection: Experimenting with Hindi and English Social Media”, MDPI, Information (2021), 12, 5.

[7] Ho-Suk Lee, Hong-Rae Lee, Jun-U Park, Yo-Sub Han, “An Abusive Text Detection System based on Enhanced Abusive and Non-Abusive Word Lists", Decision Support Systems, June (2018).

[8] Sandip Modha, Prasenjit Majumder, Thomas Mandl, Chintak Mandalia, "Detecting and Visualizing Hate Speech in Social Media: A Cyber Watchdog for Surveillance”, International Journal of Expert Systems with Applications, July (2020).

[9] Aymé Arango, Jorge Pérez, Barbara Poblete, "Hate speech detection is not as easy as you may think: A closer look at model validation (extended version)", Journal of Information Systems, June (2020).

[10] F Razali, Z Jusoh1, A SallehOmar, N Azizan, "Implementation of Anti-Profanity Words in Mobile Application Platform", IOP Conference Series: Materials Science and Engineering, International Colloquium on Computational \& Experimental Mechanics (ICCEM 2020), 1062 (2021) 012026 Doi:10.1088/1757-899X/1062/1/012026.

\subsection{Conference Proceedings}

[11]Alon Rozental, Dadi Biton, "Amobee at SemEval-2019 Tasks 5 and 6: Multiple Choice CNN Over Contextual Embedding", Proceedings of the 13th International Workshop on Semantic Evaluation (SemEval-2019) Minneapolis, Minnesota, USA, June (2019), pp 377381.

[12] Md Abul Bashar, Richi Nayak, "QutNocturnal@HASOC'19: CNN for Hate Speech and Offensive Content Identification in Hindi Language", CEUR Workshop Proceedings. Working Notes of FIRE 2019 - Forum for Information Retrieval Evaluation. Vol. 2517. Sun SITE Central Europe, Germany, pp. 237-245.

[13] Raghav Kapoor, Yaman Kumar, Kshitij Rajput, Rajiv Ratn Shah, "Mind Your Language: Abuse and Offense Detection for Code-Switched Languages", Thirty-Third AAAI Conference on Artificial Intelligence (AAAI-2019), pp. 9951-9952.

[14] Shivang Chopra, Ramit Sawhney, Puneet Mathur, Rajiv Ratn Shah, "Hindi-English Hate Speech Detection: Author Profiling, Debiasing, and Practical Perspectives", Thirty-Fourth AAAI Conference on Artificial Intelligence (AAAI-20), pp 386-393.

[15] T.Y.S.S. Santosh, K. V. S. Aravind, "Hate Speech Detection in Hindi-English Code- 
Mixed Social Media Text", CoDS-COMAD '19: Proceedings of the ACM India Joint International Conference on Data Science and Management of Data, (2019) Jan, pp 3210313.

[16] Tobias Renwick, Denilson Barbosa, "Detection and Identification of Obfuscated Obscene Language with Character Level Transforms", Proceedings of the Canadian Conference on Artificial Intelligence, June (2021), pp 1-10.

[17] Apoorva Chaudhari, Palak Davda, Monil Dand, Surekha Dholay," Profanity Detection and Removal in Videos using Machine Learning", Proceedings of the Sixth International Conference on Inventive Computation Technologies [ICICT 2021] IEEE Xplore Part Number: CFP21F70-ART; ISBN: 978-1-7281-8501-9.

[18] Prabhat Agarwal, Ashish Sharma, Jeenu Grover, Mayank Sikka, Koustav Rudra, Monojit Choudhury, "I may talk in English but gaali toh Hindi mein hi denge : A study of English-Hindi Code-Switching and Swearing Pattern on Social Networks", 9th International Conference on Communication Systems and Networks (COMSNETS) IEEE (2017), pp 554557.

[19] Meredita Susanty, Sahrul, Ahmad Fauzan Rahman, Muhammad Dzaky Normansyah, Ade Irawan, "Offensive Language Detection using Artificial Neural Network", 2019 International Conference of Artificial Intelligence and Information Technology (ICAIIT), IEEE Xplore (2019).

[20] Nurul Athirah Binti Suliman, Hazinah Binti Kutty Mammi, "Explicit words filtering mechanism on web browser for kids", 6th ICT International Student Project Conference (ICT-ISPC),IEEE, Johor, Malaysia (2017).

[21] Thomas Mandl, Sandip Modha, Anand Kumar M, and Bharathi Raja Chakravarthi, "Overview of the HASOC Track at FIRE 2020: Hate Speech and Offensive Language Identification in Tamil, Malayalam, Hindi, English and German", In Forum for Information Retrieval Evaluation (FIRE '20), December 16-20, 2020, Hyderabad, India. ACM, New York, NY, USA, pp 29-32.

[22] Sandip Modha, Prasenjit Majumder \& Thomas Mandl (2021): An empirical evaluation of text representation schemes to filter the social media stream, Journal of Experimental \& Theoretical Artificial Intelligence, DOI: 10.1080/0952813X.2021.1907792, April (2021).

[23] Michael Fell, Elena Cabrio, Michele Corazza, Fabien Gandon, "Comparing Automated Methods to Detect Explicit Content in Song Lyrics", Proceedings of Recent Advances in Natural Language Processing, Varna, Bulgaria, Sep (2019), pp 338-344.

[24] Rahul, Vasu Gupta, Vibhu Sehra, Yashaswi Raj Vardhan, "Hindi-English Code Mixed Hate Speech Detection using Character Level Embeddings", Proceedings of the Fifth 
International Conference on Computing Methodologies and Communication (ICCMC 2021) IEEE Xplore Part Number: CFP21K25-ART, June (2021), pp 1112-1118.

[25] Rahul, Vasu Gupta, Vibhu Sehra, Yashaswi Raj Vardhan," Ensemble Based Hinglish Hate Speech Detection", Proceedings of the Fifth International Conference on Intelligent Computing and Control Systems (ICICCS 2021) IEEE Xplore Part Number: CFP21K74ART; ISBN: 978-0-7381-1327-2, July (2021), pp 1800-1806.

[26] Johnny Torres, Carmen Vaca, "JTML at SemEval-2019 Task 6: Offensive Tweets Identification using Convolutional Neural Networks", Proceedings of the 13th International Workshop on Semantic Evaluation (SemEval-2019), Minneapolis, Minnesota, USA, June 67, 2019, Association for Computational Linguistics, pp 657-661.

[27] Mainak Pal, Preeti Mukherjee, Somnath Banerjee, Sudip Kumar Naskar, “JU ETCE 17 21 at SemEval-2019 Task 6: Efficient Machine Learning and Neural Network Approaches for Identifying and Categorizing Offensive Language in Tweets", Proceedings of the 13th International Workshop on Semantic Evaluation (SemEval-2019), Minneapolis, Minnesota, USA, June (2019), Association for Computational Linguistics, pages 662-667.

[28] Hui-Po Su, Zhen-Jie Huang, Hao-Tsung Chang1 and Chuan-Jie Lin2, "Rephrasing Profanity in Chinese Text", Proceedings of the First Workshop on Abusive Language Online, Vancouver, Canada, August (2017), Association for Computational Linguistic, pp 18-24.

[29] Aditya Bohra, Deepanshu Vijay, Vinay Singh, Syed S. Akhtar, Manish Shrivastava, "A Dataset of Hindi-English Code-Mixed Social Media Text for Hate Speech Detection", Proceedings of the Second Workshop on Computational Modeling of People's Opinions, Personality, and Emotions in Social Media, New Orleans, Louisiana, June ( 2018 ) Association for Computational Linguistics, pp 36-41.

[30] Puneet Mathur, Rajiv Ratn Shah, Ramit Sawhney, Debanjan Mahata, "Detecting Offensive Tweets in Hindi-English Code-Switched Language", Proceedings of the Sixth International Workshop on Natural Language Processing for Social Media , Melbourne, Australia, July (2018), Association for Computational Linguistics, pp 18-26.

[31] Faneva Ramiandrisoa, Josiane Mothe, "IRIT at TRAC 2018", Proceedings of the First Workshop on Trolling, Aggression and Cyberbullying, Santa Fe, USA, August (2018), pp 19-27.

[32] Puneet Mathur, Rajiv Ratn Shah, Ramit Sawhney, Meghna Ayyar, "Did you offend me? Classification of Offensive Tweets in Hinglish Language", Proceedings of the Second Workshop on Abusive Language Online (ALW2), Brussels, Belgium, October (2018), Association for Computational Linguistics, pp 138-148. 
[33] Zhelun Wu, Nishant Kambhatla, Anoop Sarkar, "Decipherment for Adversarial Offensive Language Detection", Proceedings of the Second Workshop on Abusive Language Online (ALW2), Brussels, Belgium, October (2018), Association for Computational Linguistics, pp 149-159.

[34] Yangfan Yao, Jian Cao, Tao Wang, Rui Liu, Zhenyuan Dai, Haoqiang Yuan, "Efficient Implementation of Dirty Words Detection in Decision Tree Model", 2020 IEEE 5th International Conference on Signal and Image Processing, pp 60-64. 